\title{
Aligning Effectiveness, Secretion of Interleukin $1 \beta$ and Pain Control During Fixed Orthodontic Treatment with Self-Ligating Appliances and Supplemental Vibrational Appliances. A Randomized Controlled Clinical Trial
}

\author{
Zamira Kalemaj ${ }^{1 凶}$, Jacopo Buti ${ }^{2}$, Andrea Deregibus ${ }^{3}$, Rosana Maria Canuto 4 , Marina Maggiora ${ }^{4}$, Cesare \\ Lorenzo Debernardi ${ }^{1}$ \\ 1. Unit of Orthodontics, Dental School, Department of Surgical Sciences, University of Torino, Italy \\ 2. School of Dentistry, University of Manchester, JR Moore Building, Oxford Road, Manchester, M13 9PL, UK \\ 3. Unit of Gnatology, Dental School, Department of Surgical Sciences, University of Torino, Italy \\ 4. Unit of Experimental Medicine and Clinical Pathology, Department of Clinical and Biological Sciences, University of Torino, Italy. \\ $\triangle$ Corresponding author: Zamira Kalemaj, Via Nizza 230, 10123 Turin, Italy; Tel: +39116687942; Email: zamira.kalemaj@edu.unito.it \\ (C) Ivyspring International Publisher. This is an open access article distributed under the terms of the Creative Commons Attribution (CC BY-NC) license \\ (https://creativecommons.org/licenses/by-nc/4.0/). See http://ivyspring.com/terms for full terms and conditions.
}

Received: 2016.09.06; Accepted: 2016.11.15; Published: 2017.01.01

\begin{abstract}
Aims: The aims of the present study were to investigate rate of mandibular incisors alignment, levels of IL- $1 \beta$ and pain and discomfort during orthodontic alignment with the following: 1) conventional-ligating appliance (CLA); 2) self-ligating appliance (SLA); 3) self-ligating appliance combined to vibrational appliance (SLA-VA).

Methods: This was a three-armed parallel group randomized clinical trial with allocation ratio 1:1:1. The main inclusion criteria were: full permanent dentition, age below 25 years, no missing teeth, first skeletal and molar class, non-extraction treatment, Little's Irregularity Index (LII) from 3mm to $6 \mathrm{~mm}$. The main outcomes were rate of mandibular incisors alignment, concentration of IL-1 $\beta$ and pain and discomfort. Measurements on models were performed at appliance placement (t0) and at monthly intervals for the first 3 months (T1, T2, T3), whereas IL-1 $\beta$ levels were measured at weekly intervals for the first 3 weeks ( $t 1, t 2, t 3)$. Differences between groups for normally-distributed variables were initially estimated through Analyses of Variance (ANOVAs) tests, followed by Post-Hoc Scheffe analysis for pair wise comparisons.

Results: Thirty-three patients were recruited and randomized with no lost to follow-up. During the first two months, faster alignment was observed for SLA $(P=0.0491)$ and SLA-VA $(P=0.0134)$ compared to CLA. At each time-point of measurement no differences were observed between SLA and SLA-VA. At $t 1$ and $t 2$ were found higher values of IL- $1 \beta$ for SLA-VA compared to SLA $(P=0.0209, P=0.0352)$ and CLA ( $P=0.0018, P=0.0029)$, whereas at $t 3$ the difference persisted only versus CLA $(P=0.0304)$. No relevant differences in terms of pain and discomfort were observed between groups at any time point. Conclusions: Self-ligating appliances seem to perform better than conventional-ligating appliances in terms of timing of teeth leveling and alignment. Vibrational devices seem to enhance secretion of IL-1 $\beta$ but do not increase effectiveness of leveling and alignment with regard to treatment acceleration or pain reduction.
\end{abstract}

Key words: Orthodontic movement, acceleration, IL $1 \beta$, pain and discomfort

\section{Introduction}

Acceleration of orthodontic tooth movement has long been desired for multiple potential benefits for both the patient and the orthodontist (1). Approaches to achieve shorter orthodontic therapies consist of attempts to enhance existing orthodontic mechanics or to apply adjunctive non-orthodontic procedures. 
The mode of archwire ligation has been considered a potentially important factor of orthodontic mechanics influencing the effectiveness of initial orthodontic alignment due to differences in frictional forces generated in the slot-wire interface (2-4). Several trials have been published comparing the self-ligating appliances with conventional ones (5-8). A recent systematic review and network meta-analysis investigating direct and indirect comparative evidence between the two ligation systems indicated non-significant difference in terms of alignment effectiveness (9). Still, results should be interpreted with caution because of the small number of studies and imprecision of the estimated treatment effect and further well-conducted studies seem to be necessary in order to estimate potential difference. Therefore, effectiveness of these appliances remains under debate.

In terms of adjunctive non-orthodontic procedures, apart from surgical approaches which are considered to be quite aggressive in terms of patients' experiences and acceptance, $(1,10)$ application of vibratory stimuli has recently gained an increasing interest. Vibratory stimuli released through non-invasive devices for few minutes per day may present a non-invasive approach which is easily accepted by orthodontic patients (11). It is reported that resonance vibration can stimulate the expression of osteoblastic cell-derived factors on osteoclast precursors like receptor activator of nuclear factor kappa B ligand (RANKL). This factor binds to its receptor, RANK, on the surface of developing osteoclastic cells resulting therefore on osteoclast formation in the PDL $(12,13)$. On the other hand, an increase of bone mass could be induced by vibration of a certain frequency, intensity and duration (13). Based on promising results of few animal studies on potential acceleratory effects of vibrations, $(12,14)$ several human studies were undertaken in order to evaluate the effect of vibrational forces on orthodontic tooth movement $(11,15-18)$. However, contrasting results emerged by different studies. Miles at al. showed that vibratory stimuli released through a vibrational appliance provided no clinical advantage for early resolution of crowding or alleviation of pain during initial alignment (11). Similarly, Woodhouse et al. found no evidence that supplemental vibrational force can increase the rate of initial tooth movement or reduce the amount of time required to achieve final alignment when used in conjunction with a preadjusted conventional appliance (15). Contrary to these findings, Pavlin et al. (19) reported that low-level cycling loading of $0.25 \mathrm{~N}$ at $30 \mathrm{~Hz}$ released by the same vibrational appliance used in the study of Miles et al. increases the rate of orthodontic tooth movement (OTM) when applied as adjunct to orthodontic treatment. In a recent study Leethankul et al. investigated through a split-mouth study the levels of IL-1 $\beta$ in gingival cervicular fluid and the rate of orthodontic tooth movement in canines stimulated by electric toothbrush compared to standard orthodontic movement of canines (16). They found enhanced secretion of IL- $1 \beta$ and accelerated OTM in canines stimulated by electric toothbrush vibrations. IL- $1 \beta$ is an inflammatory cytokine secreted as response to orthodontic tooth movement. It can stimulate osteoclast differentiation, function and survival, contributing to the activation of the bone remodeling process and tooth movement (20). While increased levels of IL-1 $\beta$ are generally related to higher pain and discomfort (13), one recent trial reported on the capacity of vibrational appliances on reducing the perception of overall and biting pain in patients undergoing orthodontic treatment (21), while another recent RCT reported that no significant differences existed in terms of pain experience between patients using conventional fixed therapy and adjunctive vibrational devices (22).

It is clear that to-date effects of various vibratory and self-ligating appliances on bone remodeling, OTM and pain control are controversial and remain unclear. The aims of the present study were to investigate rate of teeth leveling and alignment, levels of IL-1 $\beta$ and pain and discomfort at different time points during application of the following orthodontic treatments: 1) conventional-ligating appliances (CLA); 2) self-ligating appliances (SLA); 3) self-ligating appliances combined to vibrational appliances (SLA-VA).

\section{Materials and Methods}

\section{Study design}

This was a three-armed parallel group randomized clinical trial with the allocation ratio 1:1:1. No changes to the methods after trial commencement occurred. This work was reported according to the CONSORT statement (23). The procedures followed in this study were in accordance with the ethical standards of the revised Helsinki Declaration for biomedical research involving human subjects (24).

\section{Participants}

The following selection criteria were applied to patients in need of orthodontic treatment with fixed appliances: full permanent dentition (at least until first molar), age below 25 years, no missing teeth, first skeletal and molar class, non-extraction treatment, absence of diastema in mandibular arch, Little's Irregularity Index (LII)(25), ranging from $3 \mathrm{~mm}$ to 
$6 \mathrm{~mm}$, no previous orthodontic treatment, no therapeutic intervention planned involving intermaxillary or other intraoral or extraoral appliances including elastics, maxillary expansion appliances, or headgear, absence of plaque-related periodontal diseases, absence of occlusal parafunctions, absence of oral mucosal diseases, healthy periodontal status, no active caries, good systemic health, no intake of steroids/ immunosuppressants / biphosphonates, no current state of pregnancy.

Patients fulfilling the inclusion criteria were recruited and treated at the Division of Orthodontics, Dental School, University of Turin, from January 2013 to October 2015. Detailed information on each treatment and potential allocation was provided for all patients and informed consent was obtained from all of them (or parents in case of adolescents). All subjects were treated by residents in orthodontics under the supervision of specialist consultants.

\section{Interventions}

For each patient an initial standardized orthodontic case study, including study cast models, radiographs, intraoral and extraoral photographs and periodontal evaluation was performed. All patients underwent one session of oral hygiene debridement prior to appliance placement and if deemed necessary, 2-3 days before GCF samples collection. Modified Sulcus Bleeding Index prior to orthodontic therapy had to be inferior to $20 \%$. Subsequently it was constantly monitored and necessary oral hygiene sessions and instructions were given, with the aim of keeping it below $25 \%$ throughout the duration of the therapy.

MBT twin brackets 0.022-in slot (Mini-master, AO, Sheboygan, Wi USA) were used for the CLA group, whereas interactive self-ligating brackets (Empower, AO, Sheboygan, Wi, USA) for the two other groups. In the SLA-VA group the orthodontic treatment was coupled with a vibrational device (Accledent, OrthoAccel Thechnologies, Inc. Huston, USA). The following standardized archwire sequence was implemented in each case: 0.014-in thermal nickel-titanium archwire (Thermal-Ti Lite, Form I, AO, Sheboygan, Wi USA), $0.017 \times 0.025$-in thermal nickel-titanium (Thermal-Ti Lite, Form I, AO, Sheboygan, Wi USA), $019 \times 0.025$-in thermal nickel-titanium (Thermal-T D, Form I, AO, Sheboygan, Wi USA) and $0.019 \times 0.025$-in stainless steel (Form I, AO, Sheboygan, Wi USA). In the CLA group metallic ligatures were used. The vibratory device was used according to the manufacturer's indications. The device includes a biting mouthpiece that releases vibration to teeth of both lower and upper arches. Patients were instructed to use this device for 20 minutes daily, beginning from the day of appliance placement and continuing for the first 4 weeks.

\section{Outcomes}

The main outcomes were rate of mandibular incisors alignment, concentration of IL-1 $\beta$ and pain and discomfort. The secondary outcomes were quantity of GCF and periodontal depth (PD).

Rate of mandibular incisors alignment was measured on repeated cast models. All impressions were recorded using alginate and the casts were produced by mixed stone agitated under vibration. All casts were marked with an assigned sequential number. The displacement of the incisors contact points of the six mandibular incisors were quantified from each cast using LII. LII involved measuring the displacement distance from the anatomic contact point of tooth to the adjacent tooth anatomic contact point. The sum of the five displacement measures represented the relative degree of anterior irregularity. Measurements were carried out using electronic digital caliper (Mitutoyo Absolute, Tokyo, Japan) with an accuracy of $0.005 \mathrm{~mm}$. Measurements were performed on a serial of study models taken at $\mathrm{t} 0$ and at monthly intervals for the first 3 months (T1, T2, T3). The same 0.014-in round NiTi archwire was used to align the teeth throughout this period, unless replacement was needed, but the wire diameter was kept the same.

The error of the method was calculated to determine the reproducibility and reliability of the study cast measurements. Ten study models were re-measured by the same examiner at two different times, 2 weeks apart.

Apart from the first month in which several appointments were scheduled for GCF collection, during the rest of the therapy patients were seen every 4 weeks.

The levels of IL-1 $\beta$ concentration were determined using ELISA (Enzyme-Linked Immunosorbent Assay). The quantitative analyses of the GCF withdrawn were obtained by the use of an electronic analyzer (Periotron 8000, Oraflow TM, Plain view, New York, NY, USA). The Periotron measures the effect of a current of electrons on the strip soaked by the crevicular fluid and provides a given estimate of quantity of GCF.

The collection of GCF was performed immediately before application of the first archwire $(\mathrm{t} 0)$, after one hour $(\mathrm{t} 1)$, after 1 week $(\mathrm{t} 2)$ and after three weeks ( $\mathrm{t} 3)$ from the application of the first archwire. All GCF collections were performed by the same operator. 
GCF samples were assayed for the mandibular lower incisor presenting the highest displacement of contact points in both tension and compression sites, through Perio paper strips (PerioPaper, Oraflow, Plain view, New York, NY, USA). The teeth were gently washed with water, and the sites under study were isolated with cotton rolls and gently dried with an air syringe. Perio paper strips were placed into the mesio-buccal aspect of the gingival cervice until resistance was felt (approximately $1 \mathrm{~mm}$ subgingivally) and left in-situ for 30 seconds. After 60 seconds the procedure was repeated for the disto-buccal aspect of the gingival crevice. Care was taken to avoid mechanical injury. Any strips visibly contaminated with blood were discarded. The volume of GCF in the perio paper was measured with a calibrated Periotron 8000, and then the readings were converted to an actual volume (microliter) by reference to the standard curve calibrated with human serum. $(26,27)$ The paper strips were then placed in a single labeled test tube containing $350 \mu \mathrm{L}$ of phosphate-buffered saline (PBS). After 1 hour at room temperature, the strips were removed and the eluates were centrifuged $5 \mathrm{~min}$ per $6000 \mathrm{rpm}$ (Microfuge ${ }^{\circledR 22}$ R centrifuge, Beckman Coulter, CA, USA) to remove plaque and cellular elements. The samples were stored at $-80^{\circ} \mathrm{C}$ for subsequent assay. The levels of IL-1 $\beta$ were determined using commercial ELISA kits (Invitrogen, CA, USA). Fifty microliters from eluted sample were assayed according to the manufacturer's instructions. The test sensitivity is so high that it manages to detect IL- $1 \beta$ even when the quantity is $1 \mathrm{pg} / \mathrm{mL}$, as indicated in the technical cards. The amount for IL-1 $\beta$ was examined at $450 \mathrm{~nm}$ using a microplate reader (wallac 1420, PerkinElmer, Finland). Concentrations of the cytokines in each $50 \mu \mathrm{L}$ sample were determined by generation of standard curves. The IL-1 $\beta$ concentration $(\mathrm{pg} / \mu \mathrm{l})$ was calculated by dividing the total amount of IL-1 $\beta$ by the GCF volume for each sample.

The pain and discomfort was evaluated through questionnaires delivered to patients after appliance placement. Patients were asked to record their experience of pain and discomfort at 4 hours, 24 hours, 3 days, and 1 week after their visit. The subjects' discomfort experience was evaluated using a visual analog scale (VAS) including an unmarked horizontal line $100 \mathrm{~mm}$ long and weighted at both ends by the descriptive terminology "very comfortable" on the left side and "very uncomfortable" on the right (28). Each patient was asked to place a mark on the line that best corresponded to the level of pain experience. Subsequently, measurements were made of the distance from the left margin of the line to the recorded score. Considering the effect of anti-inflammatory medications on tooth movement, if patients had made use of more than 2 doses of medication during the first week after the orthodontic appointment, they had to be excluded from the study (29).

\section{Sample size}

The sample size in the present study was calculated based on published data reporting initial alignment rate of $0.05 \mathrm{~mm} /$ day, (30) with an assumed $\mathrm{SD}$ of 0.025 . We estimated the relevant expected effect on the experimental groups to be $0.08 \mathrm{~mm} /$ day. A two-tailed alpha of 0.05 and power of 0.8 suggested a minimum of 11 participants in each group. Therefore, 33 participants were recruited for the three groups.

\section{Randomization}

A list of block randomization with variable block size scheme of 3 and 6 was generated on Stata using the command "-ralloc" (StataCorp, College Station, Tex). Allocation concealment was obtained implementing a centralized assignment which did not involve trial investigators and staff. The assignment of each patient was informed through an email from the randomization center prior to the intervention. Blinding of patients and practitioner was not feasible, however outcome assessment was blind because the GCF samples, models and questionnaires were enumerated in a sequential order from the first to the last collection with no reference to pertaining patients or groups. One investigator blinded performed all measures on models. Similarly, statistical analyses were performed by a statistician on data with coded names of the groups.

\section{Statistical Analysis}

Descriptive statistics as means and standard deviation were computed at each time point for IL-1 $\beta$ concentration, rate of mandibular incisors alignment, pain and discomfort, quantity of GCF, and PD. Before performing the test of significance, normality assumptions were tested by Shapiro-Wilk test. Differences between groups for normally-distributed variables were initially estimated through Analyses of Variance (ANOVAs) tests, followed by Post-Hoc Scheffe analysis for pair wise comparisons. The effects of dichotomous or categorical baseline variables on outcomes were evaluated using multiple logistic regressions. All tests were two-tailed and all statistical comparisons were conducted at the .05 level of significance. The analysis followed the Intention to treat principle. If the number of missing values was substantial or if data was not missing at random, their effect was examined and any bias quantified. Data 
analyses were performed with Stata version 12 (Stata Statistical Software, release 12.0, StataCorp).

\section{Results}

A CONSORT diagram demonstrating subject flow through the trial is shown in Figure 1. No patient was excluded from the study and there were no lost to follow-up. There were only three missing data concerning GCF collection at $\mathrm{t} 2$ and $\mathrm{t} 3$ (one sample from the CLA group and two from the SLA group) due to non-compliance (patients did not show up at the pre-scheduled appointment). One patient assigned to SLA group was noncompliant with respect to the pain diary at $\mathrm{T} 1$. Despite of the missing data, patients were not excluded from the study because the missing data were not substantial and not dependent on the baseline parameters.

Descriptive statistics of patient allocation with gender distribution, mean age and mean pre-treatment LLI are presented in Table 1. ANOVA test was performed in order to estimate potential differences of these parameters between groups. There was no discrimination with respect to these factors between the 3 groups, validating the random assignment of appliances to each group.

Intra examiner reproducibility and reliability assessment for measurements on models showed good agreement, with intraclass correlation coefficient value of 0.96 .

Mandibular incisors alignment was mainly achieved during the two first months of therapy in all groups. LII for the baseline and the first three months of alignment is shown in Table 2. Baseline values of irregularity were similar between the three groups and no significant differences were observed for each pair wise comparison. During the first month higher alignment was observed for SLA and SLA-VA groups as compared to CLA group, differences being statistically significant, $\mathrm{P}=0.0267$ and 0.0162 respectively. Similar effect was observed at the end of the second month. The LII index was reduced significantly for the SLA and SLA-VA group, compared to CLA group. At the end of the third month almost all irregularity of mandibular incisors was solved and mild discrepancy pertained for all groups. Differences between groups were not relevant indicating comparable performance of the three therapies at the end of the third month.

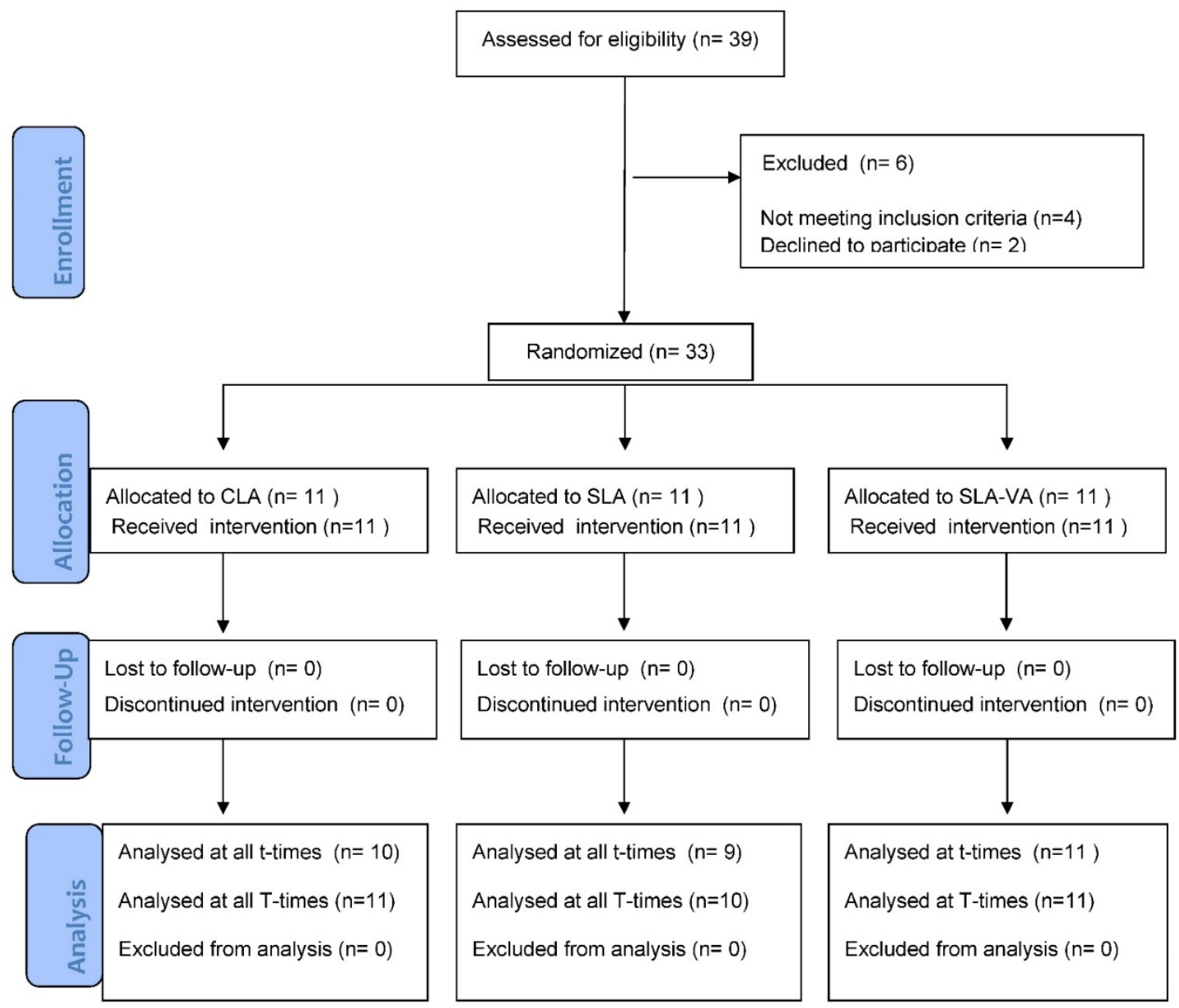

Figure 1. CONSORT diagram showing the flow of subjects in the study. 
Table 1: Characteristics of demographic parameters for patients in each group. ANOVA test for baseline differences between groups.

\begin{tabular}{llllll}
\hline & $\begin{array}{l}\text { Total } \\
(\mathbf{n = 3 3})\end{array}$ & $\begin{array}{l}\text { CLA } \\
(\mathbf{n = 1 1})\end{array}$ & $\begin{array}{l}\text { SLA } \\
(\mathbf{n = 1 1})\end{array}$ & $\begin{array}{l}\text { SLA-VA } \\
(\mathbf{n = 1 1})\end{array}$ & P-value \\
\hline $\begin{array}{l}\text { Age (mean, } \\
\text { SD) }\end{array}$ & $13.1(2.3)$ & $12.9(1.85)$ & $13.3(2.8)$ & $13.1(.07)$ & 0.8459 \\
Sex & & & & & \\
Male & 14 & 5 & 6 & 3 & \\
Female & 19 & 6 & 5 & 8 & \\
LLI (mm) & $4.70(0.68)$ & $4.52(0.83)$ & $4.82(0.40)$ & $4.75(0.70)$ & 0.0887 \\
\hline
\end{tabular}

Table 2: LII scores at different time points and Scheffe test for differences between groups. Mean and standard deviation (SD).

\begin{tabular}{lllll}
\hline \multicolumn{5}{c}{ LII $(\mathbf{m m})$} \\
\hline CLA & t0 & T1 & T2 & T3 \\
SLA & $4.52(0.83)$ & $3.24(0.63)$ & $1.56(0.40)$ & $0.45(0.08)$ \\
SLA-VA & $4.83(0.40)$ & $2.88(0.32)$ & $1.33(0.22)$ & $0.40(0.06)$ \\
P-value CLA vs. & $4.75(0.70)$ & $2.90(0.73)$ & $1.30(0.33)$ & $0.40(0.10)$ \\
SLA & & $0.0162^{*}$ & $0.0491^{*}$ & 0.0948 \\
$\begin{array}{l}\text { P-value CLA vs. } \\
\text { SLA-VA }\end{array}$ & 0.2714 & $0.0267^{*}$ & $0.0134^{*}$ & 0.0613 \\
$\begin{array}{l}\text { P-value SLA vs. } \\
\text { SLA-VA }\end{array}$ & 0.8821 & 0.9854 & 0.8752 & 0.9815 \\
\hline
\end{tabular}

Data on IL-1 $\beta$ concentration for different time points for all groups are shown in Table 3. For all the three groups the levels of cytokine reached the peak at $1 \mathrm{~h}$ after appliance placement and then started decreasing keeping high levels also after one week. At three weeks the IL-1 $\beta$ decreased progressively compared to one week but remained slightly higher than initial levels. At $t 0$ differences between groups were non-significant indicating similar distribution of IL-1 $\beta$ baseline levels as a potential effect modifier of the effects observed after therapy application. At $t 1$ the level of IL-1 $\beta$ increased markedly especially for the SLA-VA group resulting in significant difference when compared to SLA group $(\mathrm{P}=0.0209)$. The most important difference was noticed when comparing CLA and SLA-VA group $(P=0.0018)$. No statistically relevant difference was noticed between CLA and SLA at $t 1 \quad(P=0.6682)$. One week after appliance placement, differences between groups persisted. The more relevant difference remained that between CLA and SLA-VA group $(\mathrm{P}=0.0029)$. Less relevant was the difference between SLA and SLA-VA ( $\mathrm{P}=0.0352)$. Three weeks after appliance placement, differences between CLA and SLA were still not pertinent, whereas SLA-VA group exhibited higher values of IL-1 $\beta$ as compared to both SLA group $(\mathrm{P}=0.1792)$ and CLA group $(\mathrm{P}=0.0304)$.
Pain and discomfort reached the peak at 4 hours after appliance placement and decreased gradually at $24 \mathrm{~h}$ and then at 3 and 7 days for all groups. No relevant differences in terms of pain and discomfort were observed between groups at any time point of measurement. Still, SLA group exhibited lower mean values compared to CLA and SLA-VA, especially at $4 \mathrm{~h}$ and $24 \mathrm{~h}$.

Measurements of clinical variable PD indicated absence of periodontal compromised health for all patients at all times of measurement.

Regression models indicated no differences between males and females for all outcomes at all time points. Similarly, no effect of age (as categorical variable) was observed in any of the endpoint variables for all time points of measurement.

Table 3: IL-1 $\beta$ concentration levels and Scheffe tests for differences between groups in different time-points. Mean and standard deviation (SD).

\begin{tabular}{lllll}
\hline & \multicolumn{4}{c}{ IL 1B $\mathbf{( p g} / \mathbf{\mu L})$} \\
\cline { 2 - 5 } & $\mathbf{t 0}$ & $\mathbf{t 1}$ & $\mathbf{t 2}$ & $\mathbf{t 3}$ \\
\hline CLA & $25.78(4.25)$ & $41.12(7.38)$ & $33.67(9.13)$ & $28.82(6.64)$ \\
SLA & $27.00(5.24)$ & $42.94(7.81)$ & $35.80(8.85)$ & $30.11(9.13)$ \\
SLA-VA & $28.77(8.31)$ & $48.60(12.15)$ & $41.42(11.08)$ & $33.33(7.98)$ \\
$\begin{array}{l}\text { P-value CLA } \\
\text { vs. SLA }\end{array}$ & 0.6538 & 0.6682 & 0.6237 & 0.7551 \\
$\begin{array}{l}\text { P-value CLA } \\
\text { vs. SLA-VA }\end{array}$ & 0.0809 & $0.0018^{*}$ & $0.0029^{*}$ & $0.0304^{*}$ \\
$\begin{array}{l}\text { P-value SLA } \\
\text { vs. SLA-VA }\end{array}$ & 0.4086 & $0.0209^{*}$ & $0.0352^{*}$ & 0.1792 \\
\hline
\end{tabular}

Table 4: Pain and discomfort measured on a VAS scale at different time points and Scheffe test for differences between groups. Mean and standard deviation (SD).

\begin{tabular}{|c|c|c|c|c|}
\hline & \multicolumn{4}{|c|}{ Pain and discomfort (VAS 100mm) } \\
\hline & $4 \mathrm{~h}$ & $24 \mathrm{~h}$ & 3 days & 7 days \\
\hline CLA & $\begin{array}{l}58.1 \\
(14.5)\end{array}$ & $41.1(10.5)$ & $19.6(8.8)$ & $10.0(5.6)$ \\
\hline SLA & $\begin{array}{l}50.7 \\
(11.0)\end{array}$ & $36.9(7.9)$ & $17.6(5.0)$ & $8.7(4.7)$ \\
\hline SLA-VA & $\begin{array}{l}55.3 \\
(14.8)\end{array}$ & $39.1(10.5)$ & $17.1(4.8)$ & $8.6(6.3)$ \\
\hline $\begin{array}{l}\text { P-value CLA vs. } \\
\text { SLA }\end{array}$ & 0.0512 & 0.1492 & 0.3624 & 0.4379 \\
\hline $\begin{array}{l}\text { P-value CLA vs. } \\
\text { SLA-VA }\end{array}$ & 0.6257 & 0.6314 & 0.1906 & 0.3852 \\
\hline $\begin{array}{l}\text { P-value SLA vs. } \\
\text { SLA-VA }\end{array}$ & 0.3106 & 0.5916 & 0.9384 & 0.9983 \\
\hline
\end{tabular}

\section{Discussion}

This three-armed prospective randomized clinical trial investigated the effectiveness of self-ligating appliances and vibrational devices as an 
adjunct to self-ligating appliances in aligning mandibular lower incisors compared to conventional ligating orthodontic treatment. The main focus was to evaluate biological response of these orthodontic therapies in order to correlate purported acceleratory capacities to biological induced effects.

Studies in humans demonstrated that the level of IL-1 $\beta$ in GCF elevates significantly during orthodontic tooth movement. Self-ligating appliances and vibrational devices have been claimed to increase the rate of orthodontic tooth movement by accelerating periodontal and alveolar bone remodeling. In the present study the effect of self-ligating appliances and vibratory stimuli provided by vibrational device on the level of IL-1 $\beta$ in GCF was investigated. Levels of IL-1 $\beta$ in GCF resulted markedly increased one hour after appliances placement. The SLA-VA group presented significantly higher levels than the SLA group and the difference was even more pronounced when compared to CLA group.

Therefore, these results indicate that vibratory stimuli can alter the concentration of GCF components. In agreement with these findings Leethanakul et al. found higher concentration of IL-1 $\beta$ in canines after electric toothbrush application for 2 months (16). Interestingly, in their study a higher rate of maxillary canine distalization was also reported. In contrast, results of the present study indicate that the vibratory device does not influence the rate of incisors alignment during the three first months of therapy. The present results indicated an acceleratory effect in the self-ligating appliance group when compared to conventional group, but the adjunctive vibratory device does not seem to add any benefit to self-ligating therapy. Therefore, no direct positive correlation seems to underlie the relation between levels of IL-1 $\beta$ and speed of orthodontic tooth movement when using vibrational device. We hypothesized that effects of vibrations on increasing bone mineral density and structure might withstand potential effects of IL- $1 \beta$ on bone turnover. Similarly to the findings of the present study, Woodhouse et al (15) reported that the supplemental vibrational effect does not significantly increase rates of orthodontic alignment with fixed appliance.

Caution should be made while analyzing levels of IL-1 $\beta$ because of the possibility of its augmented secretion in the GCF also because of gingival irritation due to periodontal plaque-related inflammation. Although elevated levels of IL-1 $\beta$ have been related to the severity of periodontal disease, the increased concentrations of IL-1 $\beta$ observed in this study at all time points are markedly below those of patients with gingivitis and periodontitis. Moreover, values of clinical parameters $\mathrm{PD}$ and $\mathrm{BoP}$ did not change throughout the duration of the treatment, indicating absence of periodontal inflammation. Therefore, the increased IL-1 $\beta$ levels measured in this study is within the limits of an acceptable physiological response and differences in the level of IL- $1 \beta$ can be attributed solely to orthodontic tooth movement under the specific therapies.

The GCF volumes at each time point were of low statistical relevance in differences between the conventional and experimental groups, in agreement with the findings of Drummond et al. (31) and Leethanakul et al. (16) who showed that GCF volume is not a reliable biomarker of tissue remodeling during orthodontic treatment. Hence, it seems reasonable to continue using quantity of GCF only as a measurement necessary for estimating concentration of biomarkers into GCF and not for direct assumptions on orthodontic tooth movement.

A VAS was used in the current study for pain and discomfort assessment as perceived by patients. This method was selected as the measurement tool of pain and discomfort because it has been validated and used extensively in randomized trials and has shown good construct validity as compared to other pain measures (32). In the present study the pain experience at each time interval was found to be independent of bracket type or therapy. Even if the conventional group reported higher mean levels of pain, differences between groups were not relevant. This outcome confirms findings of Fleming et al. who reported that self-ligating appliances induce pain and discomfort similar to conventional appliances (33). Conversely, Miles et al. reported greater pain experience with a conventional appliance compared to passive self-ligating system (34). Authors argued that complete engagement of archwire with the conventional appliance might result in higher pain and discomfort. In the present study only patients with mild irregularity were included and full initial engagement was attempted in each case. Therefore, apart from insertion archwire facilities that self-ligating systems offer, no other benefit in terms of pain relief was observed. Similarly, it was found that supplemental vibrational devices did not induce reduced pain and discomfort. Some studies have found that vibration diminishes pain response $(21,35)$, but evidence is conflicting on this. A recent well-conducted tree-armed randomized controlled trial including eighty one subjects reported no added value of supplemental vibrational force during initial tooth alignment (17). Authors suggested that orthodontists carefully consider the use of AcceleDent as a method of pain relief or tooth movement accelerator. The findings of the present trial are in agreement with these implications, considering also 
time and cost-related aspects of supplemental orthodontic therapies.

This investigation was designed as a three-armed randomized clinical trial and recruited groups were comparable for inclusion criteria, age and sex. Still the expected effect estimated for calculating the sample size was big and therefore the number of the included patients was not large, due to a narrow range of inclusion criteria and limited number of potential confounders mainly. This accounts for a satisfactory level of internal validity. In terms of external validity, the results of this work can be mainly generalized to a population of young orthodontic patients that exhibit a moderate-to-high aligning irregularity which represent a large community of patients in everyday practice. This trial focused on the effects of therapies during the aligning phase. Therefore, the same findings can be also extended to the initial phases of the majority orthodontic treatments conducted preserving a good periodontal health of patient. Furthermore, inferences on the ability of the current investigated orthodontic therapies on accelerating orthodontic tooth movement can be made.

Crowding solving was assessed using the irregularity index measured on models. This may represent a limitation of the research, as this index is considered more appropriate for epidemiological studies rather than for clinical studies $(36,37)$ estimating changes in tooth alignment (38). Attempts were made to overcome shortcoming of this methods using a high-precision caliper and estimating previously the intra-rater reliability and reproducibility.

\section{Conclusions}

Results of the current study suggest that self-ligating appliances may perform better than conventional ligating appliances in terms of timing of teeth leveling and alignment. Vibrational devices used as adjunctive therapies seem to enhance secretion of IL- $1 \beta$ without increasing effectiveness of orthodontic therapy in terms of therapy acceleration or pain and discomfort reduction.

\section{Abbreviations}

IL 1- $\beta$ : Interleukin $1-\beta$; CLA: conventionalligating appliance; SLA: self-ligating appliance; SLA-VA: self-ligating appliance combined to vibrational appliance; PD: periodontal depth; GCF: gingival cervicular fluid.

\section{Acknowledgement}

This work was supported by the internal funds of Division of Orthodontics, Department of Surgical
Sciences, University of Turin.

\section{Competing Interests}

The authors have declared that no competing interest exists.

\section{References}

1. Kalemaj Z, DebernardI CL, Buti J. Efficacy of surgical and non-surgical interventions on accelerating orthodontic tooth movement: a systematic review. Eur J Oral Implantol. 2015 Jan;8(1):9-24.

2. Meeran NA. Self-ligating brackets: an update. J Clin Orthod. 2012 Apr;46(4):235-41.

3. Fleming PS, Johal A. Self-ligating brackets in orthodontics. A systematic review. Angle Orthod. 2010 May;80(3):575-84.

4. Chen SS-H, Greenlee GM, Kim J-E, Smith CL, Huang GJ. Systematic review of self-ligating brackets. Am J Orthod Dentofacial Orthop. 2010 Jun;137(6):726.e1-726.e18; discussion 726-7.

5. Fleming PS, DiBiase AT, Lee RT. Randomized clinical trial of orthodontic treatment efficiency with self-ligating and conventional fixed orthodontic appliances. Am J Orthod Dentofacial Orthop. 2010 Jun;137(6):738-42.

6. Celikoglu M, Bayram M, Nur M, Kilkis D. Mandibular changes during initial alignment with SmartClip self-ligating and conventional brackets: A single-center prospective randomized controlled clinical trial. Korean J Orthod. 2015 Mar;45(2):89-94.

7. Fleming PS, DiBiase AT, Sarri G, Lee RT. Efficiency of mandibular arch alignment with 2 preadjusted edgewise appliances. Am J Orthod Dentofacial Orthop. 2009 May;135(5):597-602.

8. DiBiase AT, Nasr IH, Scott P, Cobourne MT. Duration of treatment and occlusal outcome using Damon3 self-ligated and conventional orthodontic bracket systems in extraction patients: a prospective randomized clinical trial. Am J Orthod Dentofacial Orthop. 2011 Feb;139(2):e111-6.

9. Pandis N, Fleming PS, Spineli LM, Salanti G. Initial orthodontic alignment effectiveness with self-ligating and conventional appliances: a network meta-analysis in practice. Am J Orthod Dentofacial Orthop. 2014 Apr;145(4 Suppl):S152-63.

10. Hoogeveen EJ, Jansma J, Ren Y. Surgically facilitated orthodontic treatment: a systematic review. Am J Orthod Dentofacial Orthop. 2014 Apr;145(4 Suppl):S51-64.

11. Miles P, Smith H, Weyant R, Rinchuse DJ. The effects of a vibrational appliance on tooth movement and patient discomfort: a prospective randomised clinical trial. Aust Orthod J. 2012 Nov;28(2):213-8.

12. Nishimura M, Chiba M, Ohashi T, Sato M, Shimizu $Y$, Igarashi $K$, et al. Periodontal tissue activation by vibration: intermittent stimulation by resonance vibration accelerates experimental tooth movement in rats. Am J Orthod Dentofacial Orthop. 2008 Apr;133(4):572-83.

13. Huang H, Williams RC, Kyrkanides S. Accelerated orthodontic tooth movement: molecular mechanisms. Am J Orthod Dentofacial Orthop. 2014 Nov;146(5):620-32.

14. Darendeliler MA, Zea A, Shen G, Zoellner H. Effects of pulsed electromagnetic field vibration on tooth movement induced by magnetic and mechanical forces: a preliminary study. Aust Dent J. 2007 Dec;52(4):282-7.

15. Woodhouse NR, DiBiase AT, Johnson N, Slipper C, Grant J, Alsaleh M, et al. Supplemental vibrational force during orthodontic alignment: a randomized trial. J Dent Res. 2015 May;94(5):682-9.

16. Leethanakul C, Suamphan S, Jitpukdeebodintra S, Thongudomporn U, Charoemratrote C. Vibratory stimulation increases interleukin-1 beta secretion during orthodontic tooth movement. Angle Orthod. 2015 Mar 26

17. Woodhouse NR, DiBiase AT, Papageorgiou SN, Johnson N, Slipper C, Grant J, et al. Supplemental vibrational force does not reduce pain experience during initial alignment with fixed orthodontic appliances: a multicenter randomized clinical trial. Sci Rep. 2015 Jan;5:17224.

18. Rubin C, Judex S, Qin Y-X. Low-level mechanical signals and their potential as a non-pharmacological intervention for osteoporosis. Age Ageing. 2006 Sep;35 Suppl 2:ii32-ii36.

19. Pavlin D, Anthony R, Raj V, Gakunga PT. Cyclic loading (vibration) accelerates tooth movement in orthodontic patients: A double-blind, randomized controlled trial. Semin Orthod. Elsevier; 2015 Sep 9 ;21(3):187-94

20. Alhashimi N, Frithiof L, Brudvik P, Bakhiet M. Orthodontic movement induces high numbers of cells expressing IFN-gamma at mRNA and protein levels. J Interferon Cytokine Res. 2000 Jan;20(1):7-12. 
21. Lobre WD, Callegari BJ, Gardner G, Marsh CM, Bush AC, Dunn WJ. Pain control in orthodontics using a micropulse vibration device: A randomized clinical trial. Angle Orthod. 2015 Oct 23.

22. Woodhouse NR, DiBiase AT, Papageorgiou SN, Johnson N, Slipper C, Grant J, et al. Supplemental vibrational force does not reduce pain experience during initial alignment with fixed orthodontic appliances: a multicenter randomized clinical trial. Sci Rep. Nature Publishing Group; 2015 Jan 27;5:17224

23. Moher D, Hopewell S, Schulz KF, Montori V, Gøtzsche PC, Devereaux PJ, et al. CONSORT 2010 explanation and elaboration: updated guidelines for reporting parallel group randomised trials. BMJ. 2010 Jan;340:c869.

24. WMA. WMA Declaration of Helsinki - Ethical Principles for Medical Research Involving Human Subjects. 2013 Oct 19.

25. Little RM. The irregularity index: a quantitative score of mandibular anterior alignment. Am J Orthod. 1975 Nov;68(5):554-63.

26. Chapple IL, Landini G, Griffiths GS, Patel NC, Ward RS. Calibration of the Periotron 8000 and 6000 by polynomial regression. J Periodontal Res. 1999 Mar ;34(2):79-86.

27. Tözüm TF, Hatipoğlu H, Yamalik N, Gürsel M, Alptekin NO, Ataoğlu T, et al. Critical steps in electronic volume quantification of gingival crevicular fluid: the potential impact of evaporation, fluid retention, local conditions and repeated measurements. J Periodontal Res. 2004 Oct;39(5):344-57.

28. Seymour RA. The use of pain scales in assessing the efficacy of analgesics in post-operative dental pain. Eur J Clin Pharmacol. 1982 Jan ;23(5):441-4.

29. Karthi M, Anbuslevan GJ, Senthilkumar KP, Tamizharsi S, Raja S, Prabhakar K. NSAIDs in orthodontic tooth movement. J Pharm Bioallied Sci. Medknow Publications; 2012;4(Suppl 2):S304-6.

30. Pandis N, Polychronopoulou A, Eliades T. Self-ligating vs conventional brackets in the treatment of mandibular crowding: a prospective clinical trial of treatment duration and dental effects. Am J Orthod Dentofacial Orthop. 2007 Aug ;132(2):208-15.

31. Drummond S, Canavarro C, Perinetti G, Teles R, Capelli J. The monitoring of gingival crevicular fluid volume during orthodontic treatment: a longitudinal randomized split-mouth study. Eur J Orthod. 2012;34(1):109-13.

32. Haefeli M, Elfering A. Pain assessment. Eur Spine J. Springer; 2006;15 (Suppl 1):S17-24.

33. Fleming PS, Dibiase AT, Sarri G, Lee RT. Pain experience during initial alignment with a self-ligating and a conventional fixed orthodontic appliance system. A randomized controlled clinical trial. Angle Orthod. 2009 Jan;79(1):46-50

34. Miles PG, Weyant RJ, Rustveld L. A clinical trial of Damon 2 vs conventional twin brackets during initial alignment. Angle Orthod. 2006 May;76(3):480-5.

35. Marie SS, Powers M, Sheridan JJ. Vibratory stimulation as a method of reducing pain after orthodontic appliance adjustment. J Clin Orthod. 2003 Apr ;37(4):205-8-4.

36. Pandis N, Polychronopoulou A, Eliades T. Active or passive self-ligating brackets? A randomized controlled trial of comparative efficiency in resolving maxillary anterior crowding in adolescents. Am J Orthod Dentofacial Orthop. 2010 Jan ;137(1):12.e1-6; discussion 12-3.

37. Wahab RMA, Idris H, Yacob H, Ariffin SHZ. Comparison of self- and conventional-ligating brackets in the alignment stage. Eur J Orthod. 2012 Apr ;34(2):176-81.

38. Macauley D, Garvey TM, Dowling AH, Fleming GJP. Using Little's Irregularity Index in orthodontics: outdated and inaccurate? J Dent. 2012 Dec ;40(12):1127-33. 